\title{
Micropropagation of Sterile and Non-Flowering Nicotiana Lines
}

\author{
Sarala K.*, Rao R. V. S., Murthy T. G. K. and Satyavani J. V. R. \\ Central Tobacco Research Institute, Rajahmundry-533 105, India.
}

Received February 8, 2008; accepted March 9, 2008

\begin{abstract}
Summary Micropropagation could be a useful option to multiply important seeds sterile genetic stocks in crop plants. Nineteen exotic germplasm lines belonging to Nicotiana tabacum, five wild Nicotiana species and two species hybrids that did not flower and/or set seed were micropropagated in vitro through direct organogenesis using leaf explants. The plantlets thus generated were transferred to pots after hardening. All the transferred plants in germplasm accessions viz., T16, T22, T23, T26, T27, T28, T29, T30/HG, BRK 1, BRK 2, BRK 3, KRK 1, KRK 6, KRK-8, K34, K35 (T35), K36, K37 and K40 found to be male sterile. Inter subgeneric hybrid of N. gosse $\times$ N. glauca produced sterile flowers. $N$. sylvestris, and $N$. tomentosiformis plants flowered and produced seed, while $N$. arentsii, $N$. benavidesii and $N$. bonariensis plants did not flower. Thus direct organogenesis found to be useful for the in vitro multiplication of rare genotypes in genus Nicotiana. One among the twenty clones of $N$. excelsior $\times N$. plumbaginifolia hybrid transferred was found to be fertile and set seed. The progeny of fertile hybrid were all fertile. Fertility restoration in $N$. excelsiorplumbaginifolia hybrid found to be due to chromosome loss and doubling in one of the hybrid cell under tissue culture and its regeneration. Though the chromosomal change observed in the study is useful one, it suggested that care necessary to avoid such changes in other cases.
\end{abstract}

Key words Tobacco, Direct organogenesis, In vitro propagation

Tobacco is an important commercial crop and belongs to the genus Nicotiana and family Solanaceae. Out of the total 69 documented species in this genus (Goodspeed 1954, Burbridge 1960), two species viz., $N$. tabacum and $N$. rustica are cultivated throughout the world. Like in other field crops, seed production is not a problem in various Nicotiana species under normal conditions. A single tobacco plant, belonging to any of the species, produces several hundred thousand of extremely small sized seeds (Garner et al. 1936). Hence, they are commonly propagated through seeds. However, production of seed in few exotic tobacco germplasm accessions becomes a problem, as some of them are either male sterile or non-flowering under the new climatic conditions of the imported country. Also, sterility of hybrids, due to species incompatibility, is a major bottleneck in inter-specific hybrid breeding programmes. In such situations, micropropagation under in vitro is a useful option for their further multiplication and utilisation. Micropropagation technology is widely used in crops where seed production is a problem or plants regenerated from seeds are not agronomically preferred.

Central Tobacco Research Institute (CTRI), Rajahmundry, India is a premier Institute that deals with various aspects of tobacco research in India. It maintains a large collection of tobacco germplasm i.e., around 2500 accessions. During 2001-2004, it has added 122 new exotic germplasm accessions to its germplasm bank. Among them few accessions either did not flower or found to be male sterile. Meanwhile, in the inter-specific breeding programme of the Institute, two species hybrids found to be sterile. Hence, for the further utilisation of these accessions and hybrids, they were multiplied under in vitro. The present paper deals with the micropropagation at-

*Corresponding author, e-mail: sarala.ctri@gmail.com 
tempts of these sterile and non-flowering accessions/hybrids under in vitro.

Materials and methods

Plant material

During 2001-02 season, nineteen new accessions viz., T16, T22, T23, T26, T27, T28, T29, T30/HG, BRK 1, BRK 2, BRK 3, KRK 1, KRK 6, KRK-8, K34, K35 (T35), K36, K37 and K40 were imported from Zimbabwe, under the germplasm acquisition programme of CTRI. All the accessions were regenerated under the field condition. After flowering, it was found that all these lines were male sterile. As maintainer lines were not received with the accessions, seed production was not possible in these genotypes.

Among the 104 exotic Nicotiana Species accessions received during 2003-04 season, five species ( $N$. arentsii, $N$. sylvestris, $N$. benavidesii, $N$. tomentosiformis and $N$. bonariensis) did not flower. A natural hybrid of $N$. excelsior-plumbaginifolia and another hybrid, N. gossei-glauca (Murthy and Subbarao 2004) developed at CTRI were also found to be totally sterile. Therefore, in vitro direct organogenesis was attempted for their further multiplication.

\section{Culture media and incubation conditions}

MS medium (Murashige and Skoog 1962) consisted of mineral salts, vitamins, $30 \mathrm{gl}^{-1}$ sucrose and $0.8 \%$ agar supplemented with kinetin $\left(2 \mathrm{mgl}^{-1}\right)$ and IAA $\left(0.25 \mathrm{mg}^{-1}\right)$ at $\mathrm{pH} 5.8$ was used for in vitro culture. The medium was dispensed into $250 \mathrm{ml}$ culture flasks and capped with cotton plugs and autoclaved at $121^{\circ} \mathrm{C}$ for $15 \mathrm{~min}$.

For direct organogenesis, tender leaves collected from the field were surface sterilised with $0.1 \%$ mercuric chloride and cut into one $\mathrm{cm}^{2}$ bits. About four explants bits were inoculated on to $30-35 \mathrm{ml}$ culture medium in the culture flask and incubated in the culture room (physical conditions 1600 lux, $16 \mathrm{~h} \mathrm{light} / 8 \mathrm{~h}$ dark cycle, $24 \pm 1^{\circ} \mathrm{C}$ ). In-vitro generated shoots of $3-4 \mathrm{~cm}$ length were excised and again inoculated on to MS basal medium for rooting.

\section{Acclimatization}

Rooted plantlets along with flasks were initially removed and kept for one day in the media preparation room and one day in the greenhouse before transplanting to the soil. Then the plants were taken out, washed with distilled water to remove adhering culture medium and transferred to pots having sterilised soil after dipping the roots in fungicide solution. The pots were then kept inside the greenhouse under ambient conditions for one week for their establishment. Later, the pots were shifted to open space under the natural light.

\section{Characterisation of plants transferred to pots}

All the potted plants were observed for their parental characteristics and seed setting. The seed producing plants were compared with their sterile counter parts and studied their seed setting ability in the next season also. Leaf length and width, and flower length were recorded in fertile clone of $N$. excelsior-plumbaginifolia hybrid along with four sterile clones.

To study the chromosomal stability, flower buds of appropriate size were collected from fertile and sterile clones of $N$. excelsior-plumbaginifolia hybrid and fixed in acetic acid: alcohol : chloroform in 1:3:1 ratio (Modified Carnoys fixative). After $24 \mathrm{~h}$ the buds were transferred to $70 \%$ alcohol. Meiosis of the pollen mother cells was studied by smearing technique using acetocarmine stain. The slides were observed under microscope and photographed using Dig 3 camera using Dig pro. 4 software (Labomed make). 
Table 1. Response of explants of Nicotiana genotypes to tissue culture

\begin{tabular}{|c|c|c|c|c|}
\hline S. No. & Entry name & $\begin{array}{l}\text { Days taken for } \\
\text { initiation of response } \\
\text { in leaf explants on } \\
\text { organogenesis } \\
\text { medium }\end{array}$ & $\begin{array}{l}\text { Number of shoots } \\
\text { regenerated/ } \\
\text { explant }\end{array}$ & $\begin{array}{l}\text { Days taken for } \\
\text { rooting of shoots on } \\
\text { MS(b) medium }\end{array}$ \\
\hline 1. & T16 & $16-22$ & $10-12$ & 8 \\
\hline 2. & $\mathrm{~T} 22$ & $15-21$ & $8-12$ & 9 \\
\hline 3. & $\mathrm{~T} 23$ & $16-25$ & $6-10$ & 8 \\
\hline 4. & $\mathrm{~T} 26$ & $15-20$ & $10-12$ & 8 \\
\hline 5. & $\mathrm{~T} 27$ & $15-20$ & $8-10$ & 10 \\
\hline 6. & $\mathrm{~T} 28$ & $16-20$ & $7-10$ & 9 \\
\hline 7. & $\mathrm{~T} 29$ & $15-21$ & $6-11$ & 10 \\
\hline 8. & T30/HG & $15-20$ & $10-12$ & 8 \\
\hline 9. & BRK 1 & $16-20$ & $8-10$ & 10 \\
\hline 10. & BRK 2 & $15-20$ & $10-12$ & 10 \\
\hline 11. & BRK 3 & $15-21$ & $8-10$ & 8 \\
\hline 12. & K34 & $15-20$ & $10-12$ & 9 \\
\hline 13. & K35 (T35) & $16-20$ & $8-10$ & 8 \\
\hline 14. & K36 & $15-25$ & $8-10$ & 10 \\
\hline 15. & K37 & $15-20$ & $10-12$ & 9 \\
\hline 16. & K40 & $16-25$ & $8-12$ & 8 \\
\hline 17. & KRK 1 & $16-20$ & $8-10$ & 10 \\
\hline 18. & KRK 6 & $15-21$ & $10-12$ & 10 \\
\hline 19. & KRK-8 & $15-20$ & $10-12$ & 9 \\
\hline 20. & N. excelsior-plumbaginifolia & $15-20$ & $8-10$ & 8 \\
\hline 21. & N. gossei-glauca & $15-20$ & $10-12$ & 10 \\
\hline 22. & N. arentsii & $16-25$ & $8-10$ & 10 \\
\hline 23. & N. sylvestris & $15-20$ & $8-12$ & 9 \\
\hline 24. & N. benavidesii & $15-20$ & $8-10$ & 8 \\
\hline 25. & N. tomentosiformis & $15-22$ & $10-12$ & 10 \\
\hline 26. & N. bonariensis & $15-21$ & $8-12$ & 10 \\
\hline
\end{tabular}

\section{Results and discussion}

MS medium (Murashige and Skoog 1962) supplemented with $30 \mathrm{gl}^{-1}$ sucrose with kinetin $\left(2 \mathrm{mg} \mathrm{l}^{-1}\right)$ and IAA $\left(0.25 \mathrm{1}^{-1}\right)$ was earlier found to be suitable for the direct organogenesis of leaf explants of different tobacco types. Hence, the same media composition was used in the present study for the micropropagation of germplasm accessions. In all the tobacco lines, direct organogenesis started from the leaf explants in 15-25 days and about 6-12 shoots were regenerated from each explant (Table 1). Shoots of 3-4 cm length were excised and inoculated on to MS basal medium for rooting. In tobacco, MS basal medium (hormone free) found to be effective in regenerating roots from in vitro regenerated shoots and rooting started within $8-10 \mathrm{~d}$. Complete plantlets were obtained for planting under in vivo for about three months. The plantlets were transferred to pots after hardening. From each genotype, 15-20 plantlets were transferred to pots.

All the transferred plants in the 19 germplasm accessions were found to be male sterile. In vitro regenerated plants of cross $N$. gosse $i \times N$. glauca produced totally sterile flowers. In vitro rescued $N$. sylvestris, and $N$. tomentosiformis plants flowered normally and produced viable seed while $N$. arentsii, $N$. benavidesii and $N$. bonariensis plants did not flower.

Among the twenty $N$. excelsior-plumbaginifolia hybrid plants transferred, one plant was found to set seed (Fig. 1). The leaves of the fertile plant found to be shorter $(11 \mathrm{~cm})$ and broader $(6 \mathrm{~cm})$ than sterile clones $(11.5-13 \mathrm{~cm}$ and $4-4.5 \mathrm{~cm}$, respectively) (Table 2; Fig. 2). The flowers of the fertile clone were longer $(12 \mathrm{~cm})$ compared to sterile clones $(9.5$ to $10 \mathrm{~cm})($ Fig. 3). The fertile plant 


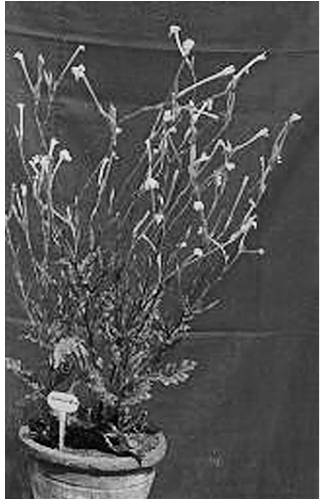

Fig.1

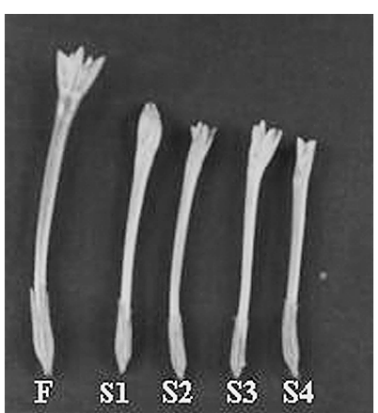

Fig. 3

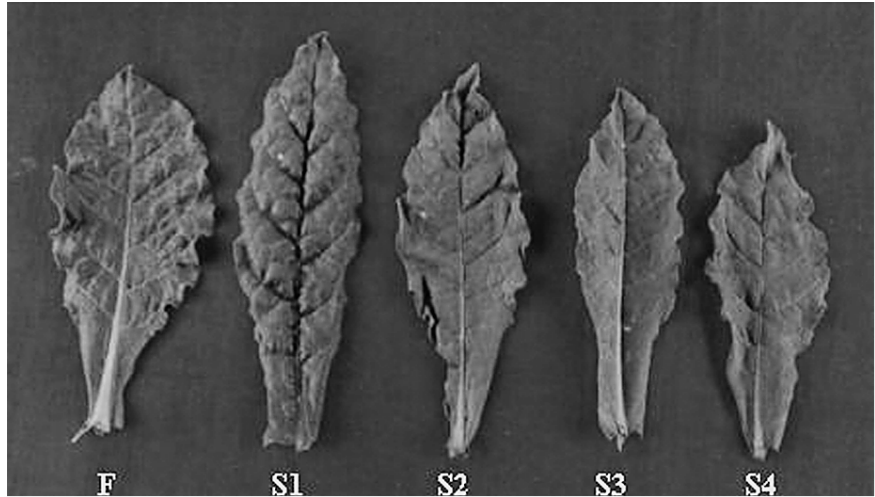

Fig.2

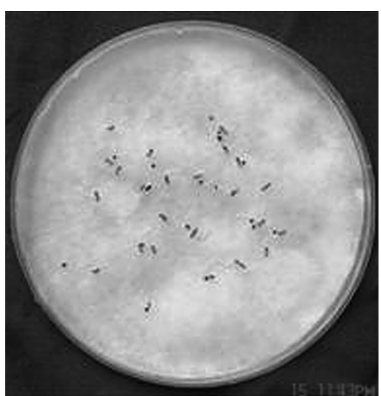

Fig.4

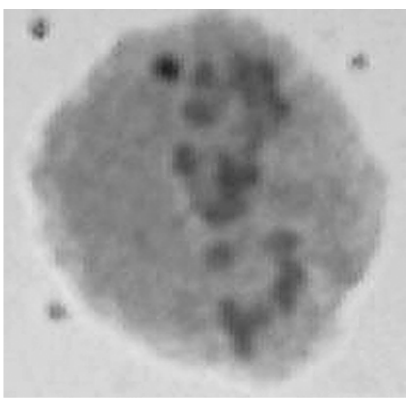

Fig. 5

Figs. 1-5. Characterisation of fertile clone of N. excelsior-plumbaginifolia, Fig. 1: Fertile clone; 2: Leaves of fertile (F) and sterile clones (S1-S4); 3: Flowers of fertile (F) and sterile clones (S1-S4); 4: Germinating seed of fertile clone and Fig. 5: 19 pair of chromosomes observed in fertile clone.

Table 2. Morphological characters of N. excelsior-plumbaginifolia plants transferred from tissue culture

\begin{tabular}{clccr}
\hline \hline S. No. & Clone & Leaf length $(\mathrm{cm})$ & Leaf width $(\mathrm{cm})$ & Flower length $(\mathrm{cm})$ \\
\hline 1. & Fertile clone & 11.0 & 6.0 & 12.0 \\
2. & Sterile clone-1 & 13.0 & 4.5 & 10.0 \\
3. & Sterile clone-2 & 12.0 & 4.5 & 9.5 \\
4. & Sterile clone-3 & 11.5 & 4.0 & 10.0 \\
5. & Sterile clone-4 & 11.5 & 4.5 & 9.5 \\
\hline
\end{tabular}

recorded $100 \%$ seed fertility (Fig. 4). Cytological analysis of the PMCs revealed normal meiotic stages in fertile clone. Chromosome number in fertile clone found to be $2 n=38$ (Fig. 5) and in sterile clones the number varied from 17-26. Under the sub-genus Petunioides, $N$. excelsior $(2 n=38)$ belongs to the section Suaveolentes and N. plumbaginifolia $(2 n=20)$ to Alatae (Goodspeed 1954, Goodspeed and Thompson 1959). The expected chromosome number in the hybrid is 29. Chromosome number of 17-26 in the sterile clones indicated loss of chromosomes due to somatic instability. Presence of 38 somatic chromosomes in the fertile hybrid may be due to chromosome doubling in a cell with 19 chromosomes and its regeneration. Laskowska and Berbeae (2005) reported variation in chromosome number among polyploidized regenerants derived from the 35-chromosome hybrid, ( $N$. tabacum cv. TB-566 tetra $\times N$. alata $) \times N$. alata. Only in one instance the number was 
70 - an exact duplication of the initial number. The lower chromosome number (from 65 to 69) in the remaining regenerants was probably due to a loss of chromosomes through somatic instability in the course of tissue culture.

All the plants raised from the seed collected from the fertile clone bred true during the next season. This indicates stability of the fertility character.

\section{Conclusion}

In the present study direct organogenesis could be successfully used for the in vitro multiplication of rare genotypes in genus Nicotiana. The study also suggested that care needed to avoid the possible chromosomal abnormalities. Fertility could be restored in N. excelsior-plumbaginifolia hybrid due to chromosome loss and doubling in one of the hybrid cell under tissue culture and its regeneration.

\section{References}

Burbridge, T. N. 1960. The Australian species of Nicotiana L. (Solanaceae). Aust. J. Bot. 8: 342-380.

Garner, W. W., Allard, H. A. and Clayton, E. E. 1936. Superior Germplasm in Tobacco. In: Year book of Agriculture; U. S. Department of Agriculture, Washington, D. C. pp 785-830.

Goodspeed, T. H. 1954. The genus Nicotiana. Chronica bot. Waltham, Mass. U.S.A.

Goodspeed, T. H. and Thompson, M. C. 1959. Cytotaxonomy of Nicotiana II. Bot. Rev. 25: 385-415.

Laskowska, D. and Berbeæ, A. 2005. Cytology and fertility of viable hybrids of Nicotiana tabacum L. cv. TB-566 with $N$. alata Link et Otto. J. Appl. Genet. 46: 11-18.

Murashighe, T. and Skoog, F. 1962. A revised medium for rapid growth and bioassays with tobacco tissue cultures. Physiol. Plant. 15: 473-497.

Murthy, T. G. K. and Subbarao, I. V. 2004. Some new interspecific hybrids in the genus Nicotiana-characterisation and utilisation. Tob. Res. 30: 33-41. 